\title{
Construction and effectiveness of vomit-free ward ${ }^{\dagger}$
}

Received: 3 August 2018; Accepted: 23 November 2018; Published: 20 September 2019

Abstract: Objective: To build a vomit-free ward of scientific and standardized management mode, and to evaluate its clinical effects on preventing nausea and vomiting in patients undergoing chemotherapy.

Methods: A total of 200 patients suffering from nausea and vomiting were randomly selected from the registration system, from which 120 cases were enrolled according to the inclusion and exclusion criteria. These subjects were divided into control group and observation group (60 patients in each). Both of the two groups received routine nursing care, while the treatment group subjects lived in the vomit-free wards where extra nursing interventions were applied to prevent chemotherapy-induced nausea and vomiting (CINV), with constant observation of the frequency and severity of nausea and vomiting. The outcomes of the subjects from both the groups as well as nurses' cognition level of CINV were compared after four chemotherapy cycles.

Results: The difference in the scoring for the frequency and severity of nausea and vomiting in two groups was statistically significant $(P<0.05)$. The scores of nurses' cognition level of CINV were compared between before and after the interventions, and the difference had statistical significance $(P<0.05)$.

Conclusion: The management mode aiming to build vomit-free wards could decrease the incidence of CINV in patients during chemotherapy, and at the same time increased the cognitive level of nursing staff toward CINV, which is of great value in clinical practice and is worthy of further application.

Keywords: tumor $\bullet$ nausea and vomiting $\bullet$ no vomiting ward $\bullet$ nursing $\bullet$ chemotherapy $\bullet$ antiemetic

(c) Shanxi Medical Periodical Press.

\section{Introduction}

Chemotherapy-induced nausea and vomiting (CINV) is a common adverse reaction during chemotherapy. Nausea and vomiting can cause mental stress, loss of

${ }^{\dagger}$ This project was supported by Southern Hospital of Southern Medical University (No. 2016H006). appetite, and poor sleep. It will have a significant negative impact on the patient's emotion, social abilities, and other aspects. ${ }^{1}$ Patients with tumors need long-term treatments by phases, which requires good physical affordability to chemotherapeutics and large intake of nutrients. As a side effect of chemotherapy, patients can suffer hair loss, weakened immunity, and loss of appetite. Frequent nausea and vomiting can lead to

How to cite this article: Yao ZQ, Wang S, Zhou XP, Luo YL, Ao Q, Miao JX. Construction and effectiveness of vomit-free ward. Front Nurs. 2019; 3: 183-190. 
electrolytes' disturbances, reduce the effectiveness of treatment, and make patients suffer self-image disorder and anticipatory grieving. ${ }^{2}$ In some worst cases, it will affect the doctor-patient relationship and cause medical disputes, resulting in patient's termination of the treatments. ${ }^{3}$ In this way, the prevention of the occurrence of CINV effectively has become a burning problem to be settled. According to our department condition and the results of previous studies, we have set up a scientific and standardized management model for vomit-free wards, of which influences are significantly positive and the report is as follows.

\section{Materials and methods}

\subsection{General information of patients}

Random sampling was used in selecting 200 patients undergoing chemotherapy in our hospital from April to October 2017. According to the inclusion and exclusion criteria, 120 patients were recruited. Among those 80 patients excluded, 53 were severely malnourished and could not accept the chemotherapy; 3 patients had suffered advanced lung cancer; 8 patients had refractory vomiting; 7 patients had a Karnofsky functional state scoring standard (KPS) $)^{4}$ score of $<50$; and 6 patients had severe myelosuppression. The family members of the remaining three patients did not agree with the intervention method, so 120 cases were eventually included. By the use of random number table, patients were divided into control group and observation group with 60 cases in each. There were 55 cases of lung cancer, 30 cases of cervical cancer, 16 cases of gastric cancer, and 19 cases of breast cancer. There was no significant difference in gender, age, tumor stage, education level, and marital status between the two groups $(P>0.05)$.

\subsection{Inclusion and exclusion criteria}

Inclusion criteria are as follows: (1) There was no significant difference in the dosage of chemotherapeutic agents and the severity of their adverse reactions during chemotherapy between the two groups $(P>0.05)$. (2) There was no significant difference in body affordability $(P>0.05)$ and no contraindication to chemotherapy. (3) KPS score $\geq 60$, Eastern Cooperative Oncology Group (ECOG/ZPS) scores $\leq 2$. (4) Compliance to the therapy and the treatment. (5) Not less than four chemotherapy cycles due, and the expected survival time $\geq 6$ months. (6) Voluntary participation with informed consents.

Exclusion criteria are as follows: (1) lack of compliance or dropout; (2) obstinate vomiting ${ }^{5}$; (3) complicationcombined like severe myelosuppression, serious infection, liver and kidney failure, mental illness, gastrointestinal perforation, etc.; and (4) poor understanding or impaired communication.

\subsection{Construction and management of vomit-free ward}

\subsubsection{Build a management team and specify the job assignments}

Fourteen medical staffs from our department were recruited to the management team, aged 23-51 $(29.80 \pm 6.13)$ years; length of service: $2-33$ years; education level: three college graduates, eight bachelor degree holders, three doctoral degree holders; technical title: three nurses, four nurse practitioners, two doctors, two nurses-in-charges, one associate professor of nursing, one professor of nursing, one professor of treatment; marriage: six married and eight unmarried. Head of department and head nurse served as the team leaders to supervise the implementation of the work. The doctors in charge and nurses in charge took the main responsibilities for preventing vomiting and managing patients who suffer from nausea and vomiting. Both doctors and nurses were involved in the assessment of antiemetic treatments and discussed the antiemetic formulation together. They carried out health education on patients, evaluated the effects of the treatments on a regular basis, and tracked the patients throughout the whole process. Patients and their families were invited to participate in the discussion about antiemetic formulation too, and studied the self-gradation of nausea and vomiting as well as some other related knowledge, so that they were able to report their own conditions to doctors in charge and nurses in charge a timely manner. By doing those, an integrated, systematic, and standardized management model was built ultimately.

\subsubsection{Staff training and appraisals}

With reference to the US version of the vomiting prevention and treatment guideline for cancer treatment in $2004,{ }^{6}$ the vomiting prevention and treatment guideline for Chinese cancer treatment (2014 version), ${ }^{7}$ as well as the nursing experience for CINV from home and abroad, the head of department and head nurse were in charge of training team members with professional and systematic theoretical knowledge, based on the patient's own situation and the current practical experience of our department. The training contents include the assessment methods for CINV risking factors; the mechanism and adverse reactions of common antiemetic drugs ${ }^{8}$; the gradation of vomiting caused by chemotherapeutic drugs and the categories of antiemetic drugs ${ }^{9}$; and 
methods to conduct effective education. The training was conducted through lectures, case discussions, and nursing ward rounds. The effectiveness of the training was evaluated via theoretical knowledge tests on CINV risks assessment, mechanism of CINV, application of antiemetic drugs, etc.

\subsubsection{Standardized management of CINV}

After the establishment of a no-vomiting team, all the members got the specified assignment. The observation group was given the standardized management of CINV when they were in the hospital, including the management before, during, and after the chemotherapy. The specific process is shown in Figure 1.

\subsection{Methods}

\subsubsection{Control group}

The control group was given routine nausea and vomiting care, including: (1) guidance on daily diet and medication; (2) prevention and measures to CINV; (3) social-emotional support and stress-coping strategies; (4) family care guidance and disease counseling; and (5) decent ward environment, which satisfied patients' living needs.

\subsubsection{Observation group}

\subsubsection{Assessment of CINV risk factors and levels of chemotherapy-induced vomiting}

According to the chemotherapy regimen, we made a characterized assessment form for CINV risk factors, in terms of the age, gender, alcohol intake, previous motion sickness, previous pregnancy and pregnancy reactions, previous vomiting experience, emotional conditions, etc. Patients who had two or more factors mentioned above would be considered as vulnerable ${ }^{10}$ and then a reminder table mat should be posted on the desks of their charge doctors. Similarly, the patients' headboards would be marked with a sign to indicate that they were in high risks. Referring to the National Comprehensive Cancer Network of the United States (NCCN) clinical guidelines (2014 version), ${ }^{6}$ we made an assessment to the level of emesis caused by chemotherapeutics and determined which grade of risks patients had, so that we were able to take full prevention of relevant conditions.

\subsubsection{Create specialized archives for CINV}

All patients in the observation group had CINV archives created by nurses in charge before chemotherapy, which included general information, occurrence, duration and severity of nausea and vomiting, category and dosage of medication, time of administration, nursing care, and efficacy evaluation along with the previous history concerning nausea and vomiting. These details were recorded and assessed at regular intervals by nurses. When patients were about to leave the hospital, their families or themselves would be taught about when and how to do the record, by which doctors and nurses could constantly observe the effects of the treatments when they were admitted to hospital next time.

\subsubsection{Proper use of antiemetic drugs}

Before the chemotherapy, assessment of high-risk factors of CINV and the severity of vomiting should be accomplished to make relevant preventive plans. For example, prophylactic antiemetic therapy should be taken ahead of chemotherapy. Patients undergoing chemotherapy by using of drugs with high and moderate emetic risks might be possible to suffer CINV unless after at least 2 or 3 days later. Therefore, preventions were supposed to be implemented during the whole risk period. After the patients were discharged from the hospital, they should be followed up regularly to facilitate the reassessment when they were admitted next time.

\subsubsection{Non-pharmacological management in chemotherapy}

All patients were instructed for daily life and were informed of the physical influences of CINV and the significance of anti-emesis. They were advised to have light diet of nutritional balance and more fresh fruits and vegetables for healthy bowel movements. The daily intake of water was ensured to be above $2000-3000 \mathrm{~mL}$ during the treatment period. Patients' needs would be met to the most degree and they could receive psychological support.

\subsubsection{Continuing nursing care for CINV patients}

If nausea and vomiting were well-controlled and there was no other discomfort when treatments finished, the doctors in charge would give patients discharge instructions. Nurses in charge were expected to give lectures to patients on how to control nausea and vomiting with CINV management mode, and inform them to do records in time after they get discharged from hospital, such as the nature, quantity, color of vomitus, and its attack time. They also should be taught to write diaries about their appetite and daily activities, which would be 


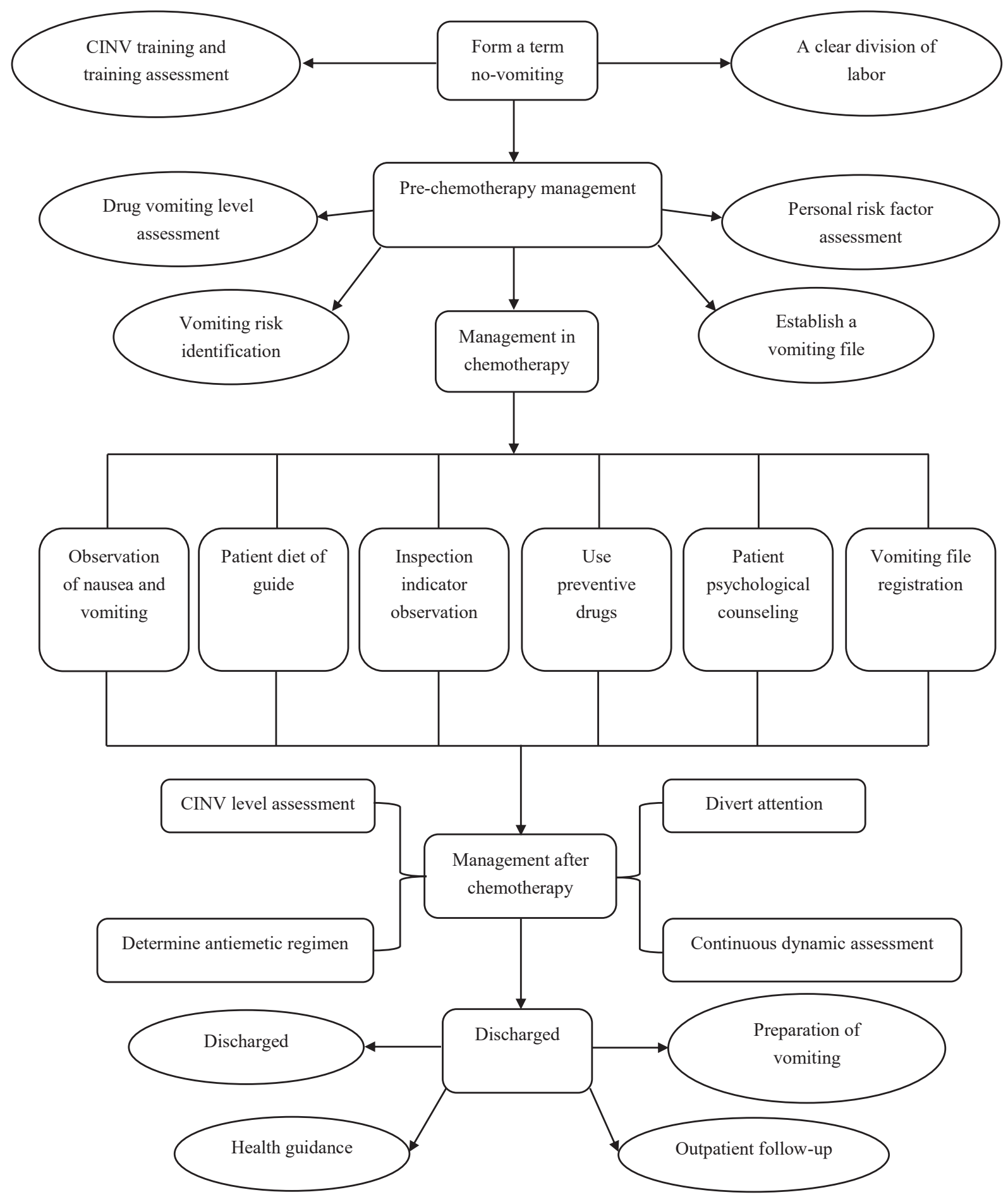

Figure 1. Standardized management of CINV.

a good reference for doctors and nurses for the next time for admission. Constant telephone follow-ups was offered to patients too, which included the patients' daily conditions, the degree of nausea and vomiting, eating conditions, and coping ability. These details were collected in the medical management system and transferred to the doctors in charge in a timely manner. Doctors and nurses would discuss on the antiemetic plan, or make adjustments according to the patients' condition if necessary. 


\section{Evaluation of the effectiveness of the management mode for vomit-free ward}

\subsection{Nurses' cognitive level of CINV before and after training}

The CINV training manual was designed by the research team, including general knowledge of the adverse reactions of chemotherapeutics, pathophysiology, and assessment methods for nausea and vomiting, and relevant medical interventions. There were 15 questions, scoring 15 points in total. All the staff involved should participate in theoretical knowledge tests and their scores would be recorded by questionnaires. They had to fill in the questionnaire separately and hand it on the spot.

\subsection{The frequency and degree of nausea and vomiting in patients}

At present, the United States National Cancer Institute's adverse event evaluation criteria ${ }^{11}$ (NCI-CTCAE, V4.03) are commonly used throughout the world. The nausea severity was classified into four levels: Level 0: no nausea; Level 1: less appetite, but no change in eating; Level 2: nutritional deficiency, decreased oral intake; Level 3: severe nutritional deficiencies and requiring gastrointestinal nutritional support; among them, the appetite and nutrition assessment criteria were judged by Guigoz's Micronutrition Assessment Form (MNA), ${ }^{12}$ which was developed in 1996. The scale includes four dimensions: anthropometric, overall assessment, dietary assessment, and subjective assessment with 18 items covered. Total scores range from 0 to 30 points, $\geq 24.0$ for good nutrition, 17.0-23.5 for risk of undernutrition, $\leq 17.0$ for undernutrition, Cronbach's a coefficient of the scale is 0.811 . The criteria for vomiting contain five levels: Level 0: no vomiting; Level 1: one to two episodes within 24 hours (interval of 5 minutes); Level 2: three to five episodes within 24 hours (interval of 5 minutes); Grade 3: $\geq 6$ episodes within 24 hours (interval of 5 minutes); Level 4: life-threatening and needing urgent treatment.

\subsection{Statistical method}

The data obtained were statistically analyzed by use of SPSS 20.0. The measurement data were described by $\mathrm{M} \pm \mathrm{SD}$ and analyzed through the $t$-test. The ordinal data were tested by the Wilcoxon rank sum test. $P<0.05$ was considered statistically significant.

\section{Results}

The scores of CINV knowledge before and after nurse training are shown in Table 1.

The frequency and degree of nausea and vomiting in both groups are shown in Tables 2 and 3.

\section{Discussion}

\subsection{Standardized management mode of vomit-free wards raised nursing staff's awareness of CINV}

According to Table 1, the CINV knowledge scoring was $(70.47 \pm 9.19)$ points and $(98.72 \pm 9.84)$ points, respectively, before and after the scientific training, and the difference was statistically significant $(t=7.851$, $P<0.001)$, which indicates that the implementation of vomit-free ward care mode can effectively raise the nursing staff's awareness of CINV.

\subsection{Standardized management mode of vomit-free wards reduces the incidence of CINV among patients}

In this study, the frequency and degree of nausea and vomiting were significantly decreased after building

\begin{tabular}{lccccc}
\hline Groups & Quantity & 0 level & -level & $\alpha$ level & $\beta$ level \\
\hline \hline Control group & 60 & 5 & 14 & 33 & 8 \\
Observation & 60 & 24 & 22 & 12 & 2 \\
group & & & & & \\
$Z$ & & -2.151 & & \\
$P$ & & 0.031 & \\
\hline
\end{tabular}

Table 2. Comparison of the frequency and degree of nausea after the interventions.

\begin{tabular}{lccccc}
\hline Time & Quantity & $\begin{array}{c}\text { Risk of vomiting } \\
\text { evaluation }\end{array}$ & $\begin{array}{c}\text { CINV occurrence } \\
\text { mechanism }\end{array}$ & $\begin{array}{c}\text { Comprehensive application of } \\
\text { antiemetic drugs }\end{array}$ & Total score \\
\hline \hline Before training & 14 & $41.00 \pm 3.75$ & $11.74 \pm 2.12$ & $17.73 \pm 3.32$ & $70.47 \pm 9.19$ \\
After training & 14 & $52.14 \pm 4.20$ & $14.36 \pm 2.00$ & $32.22 \pm 3.64$ & $98.72 \pm 9.84$ \\
$T$ & & 7.403 & 3.364 & 11.005 & 7.851 \\
$P$ & & $<0.001$ & $<0.01$ & $<0.001$ & $<0.001$ \\
\hline
\end{tabular}

Table 1. Score comparison of chemotherapy-induced nausea and vomiting (CINV) knowledge before and after nurse training. 


\begin{tabular}{lccccc}
\hline Groups & Quantity & 0 level & - level & $\alpha$ level & $\beta$ level \\
\hline \hline Control group & 60 & 11 & 18 & 15 & 7 \\
Observation group & 60 & 33 & 16 & 3.891 & \\
$Z$ & & & 0.000 & \\
$P$ & & & & \\
\hline
\end{tabular}

Table 3. Comparison of the frequency and degree of vomiting after the interventions.

vomit-free wards (Tables 2 and 3), indicating that the management mode of vomit-free wards had certain positive effects and practical value. Table 2 shows that the two groups of patients in terms of their control of nausea, the difference of the incidence of level I nausea was less prominent than others, but was still statistically significant. The main reasons for this difference were (1) nausea is the most common adverse reaction of patients in chemotherapy and can be reduced to the most degree, but for most patients, it cannot be completely avoided and (2) before the chemotherapy, all patients were evaluated with $\mathrm{NCI}-\mathrm{CTCAE}$ scale. If the nausea patients suffering reached grade III and if their vomiting reached grade IV, conservative treatment would be considered. The nausea of patients with level II or III got reduced to level I, which increased the number of patients with level I to some extent, as the vomiting condition in Table 3 . With the advancement of medical technology, the quality of life among cancer patients has become a widespread concern for medical staff. Especially nausea and vomiting are tremendously harmful to cancer patients. Therefore, the establishment of vomit-free wards cannot be delayed. The cognitive level of China's health care workers toward the knowledge of preventing CINV is in great need to be improved. ${ }^{13}$ However, there are few domestic reports available. It is recommended that we should refer to domestic and foreign relevant guidelines and

\section{References}

1. Detterbeck F, Tanoue L, Reid A. Correspondence. J Natl Compr Canc Netw. 2013; 11: 365-366.

2. Kaushal P, Atri R, Soni A, Kaushal V. Comparative evaluation of triplet antiemetic schedule versus doublet antiemetic schedule in chemotherapy-induced emesis in head and neck cancer patients. Ecancer medical science. 2015;9:567.

3. So WK, Chan DN, Chan HY, et al. Knowledge and practice among Hong Kong oncology nurses in the management of chemotherapy-induced nausea and vomiting. Eur J Oncol Nurs. 2013;17:370-374.

4. Li Q, Xu P, Xiong M. Effect of integrated Chinese and Western medicine on the efficacy, side effects anti-emesis experience from other countries, ${ }^{14,15}$ put this management mode into wide practice, according to the current medical situation in China. In that case, health care workers will be given more training related to the knowledge of CINV preventions, so as to establish a standardized and systematic anti-vomiting team. There are various tools for vomit-free assessment in China, but it lacks unified standard. It is advised to focus on the adjustment of management mode and to create practical assessment tools for clinical use. Mainstream hospitals should actively organize expert teams for teaching vomit-free knowledge as soon as possible and to set up a practical team for anti-vomiting. Another point is, we must pay more attention to no-vomit instructions to patients and their families, in order to improve their quality of life, and promote the development of China's health care services.

\section{Ethics approval}

This study was approved by the ethics committee of Southern hospital (IRB approval number: NFEC201908-Y2-06). Written informed consent was obtained from all of the experts.

\section{Conflicts of interest}

All contributing authors declare no conflicts of interest. and KPS score of patients with non-small cell lung cancer. Modern J Integr Traditional Chinese Western Med. 2019; 28: 991-993 (in Chinese).

5. Renard D, Castelnovo G, Bories C, Rieux C, BernardDecot C. Paraneoplastic rhombencephalitis and cerebellitis preceded by isolated intractable vomiting. Acta Neurol Belg. 2017;117:741-742.

6. NCCN. Clinical Practice Guidelines in Oncology: Antiemesis. http://www.Nccn.org/professionals/ physician_gls/pdf/antiemesis.pdf. Accessed 25 February 2014.

7. China Anti-Cancer Association Cancer Rehabilitation and Palliative Care Committee (CRPC), China Society 
of Clinical Oncology Anti-Tumor Drug Safety Management Expert Committee (ASMC). Tumor treatment related vomiting prevention guidelines (2014 edition). J Clin Oncol. 2014;19: 263-273 (in Chinese).

8. Zhang HA, Zong BB, Jia ZL. Selection of antiemetic regimen in combination with opioids and high emetogenic chemotherapy drugs in patients with cancer. Primary Med Forum. 2017;21: 4979-4981 (in Chinese).

9. Cao J. Analysis of the use of different antiemetics in 595 patients with cancer during chemotherapy. J Contemporary Med. 2018;16:158-160 (in Chinese).

10. Tan L, Liu XY, Wei D, et al. Establishment and effectiveness of nursing work mode for lung cancer chemotherapy without vomiting ward. China Nurs Manage. 2017; 02:244-248 (in Chinese).

11. Luo L. Clinical study on the treatment of nausea and vomiting caused by platinum-containing chemotherapy with auricular acupoints. Guangzhou: Guangzhou University of Chinese Medicine. 2011:6-10 (in Chinese).

12. Geng AJ, Zhang LP. Observation on the efficacy of ginger combined with ondansetron in preventing vomiting in patients with chemotherapy. Nurs Rehabil. 2008;7:880-881 (in Chinese).

13. Tian C, Su YH, Qiang WM, et al. Investigation on the nursing status of nursing nausea and vomiting nurses in oncology nurses. J Nurs Sci. 2015;30:1-4 (in Chinese).

14. Spruyt OW. Ondansetron to prevent vomiting after cancer chemotherapy. Med Lett Drugs Ther. 1991;33:63-64.

15. Dzamic A, Vilotic J, Radovanovic M. Administration of water before giving chloroform to prevent vomiting. Lancet. 1905;165:178. 